\title{
Optical Solitons in Fibres
}

\author{
E.M. Dianov \\ General Physics Institute, \\ Russian Academy of Sciences, Moscow
}

Solitary waves propagating for long distances in glass fibres without changing their shape may provide a globe-spanning soliton-based optical communications system within a decade.

In dispersive, nonlinear, weakly attenuating media there may exist a special class of so-called solitary waves which can propagate for long distances without changing their shape. Such a solitary wave was first reported in 1834 by the Scottish engineer Scott Russell. He observed that the wave in the shape of a large solitary hump generated by a barge moving along a narrow channel propagated without a noticeable change to the shape or a reduction in the velocity. Scott Russell subsequently reproduced the wave in the laboratory and named it a "solitary wave". Theoretical confirmation was made in 1895 by Korteveg and de Vries when they obtained the equation of wave propagation along the surface of water in a shallow channel.

If the shape of solitary waves does not change at their collision they are called solitons, a name given by Zabusky and Kruskal in 1965, thus emphasising the particle-like behaviour during collisions.

Solitons are no doubt widely found in nature and are intensively studied in many branches of physics (hydrodynamics, plasma, laser physics, etc.). In the case of optical solitons in fibres, in addition to the fundamental interest there also exist important applications in optical fibre communications and signal processing.

\section{Optical Fibres as a Medium}

Before attempting to better understand the great interest in optical solitons in fibres one should go back to 1960 when the laser first appeared. One of the initial experiments where lasers were used concerned data transmission through the atmosphere. Using optical waves as carriers made it possible, in principle, to transmit information at rates many orders of magnitude higher than for radiowaves.

Professor E.M. Dianov is presently the Deputy Director of the General Physics Institute of the Russian Academy of Sciences, 38 Vavilov St., USSR-117942 Moscow GSP-1, where he also heads the department for fibre optics. He graduated from the Moscow State University in 1960 and received his Ph.D from the P.N. Lebdev Physical Institute in 1966, where he remained on the staff until joining the General Physics Institute in 1982.
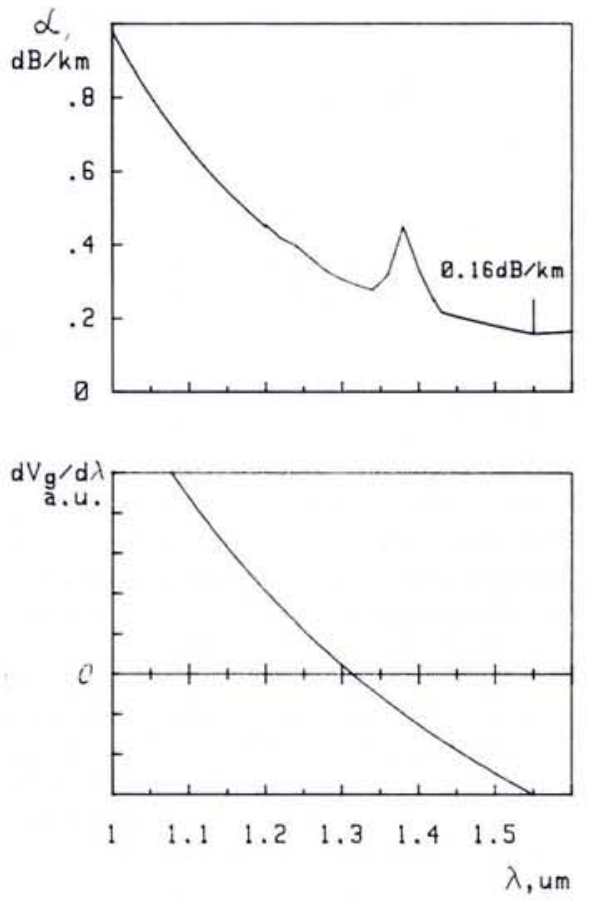

Fig. 1 - Silica glass optical fibres. a, upper) Wavelength dependence of single-mode fibre losses: there is a minimum loss of 0.16 $\mathrm{dB} / \mathrm{km}$ near $1.55 \mu \mathrm{m}$.

$b$, lower) Wavelength dependence of $d V_{g} / d \lambda$, the group velocity dispersion: note the change of sign at approximately $1.3 \mu \mathrm{m}$.

However, the first experiments showed that the atmosphere, owing to meteorological effects, is not a reliable medium. The search for a transmitting medium which isolates light from atmospheric instabilities brought about in the 1970's the development of optical glass fibres with low optical losses.

It looked at first as if obtaining losses below $1 \mathrm{~dB} / \mathrm{km}$ in the near-infrared region would make possible the transmission of information at rates of tens of Gbit/s over distances of hundreds of kilometres without repeaters. A revolution in communications technology might have arisen as a consequence.

However, while shifting from the free atmosphere to glass fibres as the transmitting medium eliminated certain difficulties related to instabilities, two new problems, inherent to condensed media, limited data transmission.
The first problem, connected with the attenuation of light in optical fibres, was solved by using chemical vapour deposition processes for fibre fabrication. Silicabased optical fibres with close to theoretical losses were obtained. They exhibit a minimum loss of about $0.16 \mathrm{~dB} / \mathrm{km}$ at wavelengths $\lambda \approx 1.55 \mu \mathrm{m}$ (Fig. 1a) equivalent to an attenuation coefficient of $\lambda$ of $\approx 4 \times 10^{-7} \mathrm{~cm}^{-1}$ or a halving of the intensity of optical radiation over $\approx 18 \mathrm{~km}$.

The second problem is the dependence of the pulse propagation velocity on the light frequency (group velocity $V_{\mathrm{g}}$ dispersion - GVD) which is connected with the frequency dependence of the refractive index of glasses. As optical pulses have a finite spectral width, GVD leads to a temporal pulse broadening that limits the transmission rate to hundreds of Mbit/s. From Fig. 1b showing the wavelength dependence of GVD for silica glass we conclude, first, that GVD equals zero at $\lambda \approx 1.3 \mu \mathrm{m}$. Secondly, GVD changes sign: it is positive for $\lambda<1.3 \mu \mathrm{m}$ but negative for $\lambda>1.3 \mu \mathrm{m}$. As will be demonstrated below, the negative GVD (i.e., $\partial V_{g} / \partial \lambda<$ 0 ) is a necessary condition for soliton propagation in fibres. It is important to note that for silica fibers the region of minimum losses $(\lambda \approx 1.55 \mu \mathrm{m})$ lies in the negative GVD spectral region.

On the basis of the spectral dependence of GVD one can conclude that it is possible to achieve high data transmission rates by tuning the laser source to the wavelength at which GVD passes through zero $(1.3 \mu \mathrm{m})$. Such systems have been developed and find widespread application. But there is another, more promising, way of overcoming the effect of GVD on the transmission rate: the combination of nonlinearity and negative GVD results in optical soliton pulse propagation (i.e., without shape change). Soliton propagation in fibres was recognised theoretically by $A$. Hasegawa and $F$. Tappert in 1973: it was observed experimentally seven years later when suitable lasers as well as optical fibres with low losses at $\lambda \geq$ $1.3 \mu \mathrm{m}$ became available.

\section{Nonlinearity of Silica Glass Fibres}

To gain insight into soliton effects in fibres, consider briefly the optical nonli- 
nearity of glasses. Optically nonlinear materials show changes in their refractive index if placed in electric, magnetic or optical fields. The application of a short, high-intensity optical pulse to a molecule results in an intensity-dependent refractive index. Two, distinct, physical contributions to the nonlinear refractive index arise. There is an electronic contribution from the nonlinear distortion of the electronic structure of the molecule. This component responds rapidly $(<1 \mathrm{fs}$ where $1 \mathrm{fs}=10^{-15} \mathrm{~s}$ ) to field changes and can be considered instantaneous.

A nuclear contribution arises from an optical field-induced change in the motions of the nuclei that cannot respond rapidly to field changes (the delay time is of order $100 \mathrm{fs}$ ). It is important to note here that the delayed nonlinear response associated with the excitation of molecular vibrations gives rise to Raman effects.

Consider first relatively long optical pulses (pulse width $T_{0}>1 \mathrm{ps}$ where $1 \mathrm{ps}$ $\left.=10^{-12} \mathrm{~s}\right)$. Both contributions to the change of the refractive index may be considered as instantaneous, so the refractive index $n$ of a glass fibre depends on the laser radiation intensity $I$ as $n=n_{0}+n_{2} I$ where $n_{0}$ is the refractive index at an arbitrarily low intensity, $n_{2}$ is a constant with numerical value $3.2 \times 10^{-16} \mathrm{~cm}^{2} / \mathrm{W}$ for silica glass. Silica glass is not a good nonlinear material and the low value of $n_{2}$ confirms this. But whenever the glass is used in the form of a fibre, even a small nonlinearity produces a large effect. This is connected with the small cross-sectional area $\left(10^{-6} \mathrm{~cm}^{2}\right)$ of the core of singlemode fibres. For if the power of the input pulse is $1 \mathrm{~W}$, the intensity is $1 \mathrm{MW} / \mathrm{cm}^{2}$. Moreover, the high intensity level is maintained at an almost constant value along the whole interaction length, which is limited only by the fibre loss and can be as long as several kilometres. This feature allows the exchange of length for power, permitting the observation of nonlinear phenomena at powers many orders of magnitude lower than in bulk materials.

\section{Self-phase modulation}

The intensity dependence of the refractive index of fibres leads to the phenomenon of self-phase modulation. This phenomenon is basically very simple. Consider an optical pulse with the intensity envelope $|E(t)|^{2}$ propagating along a fibre of length $L$ during a time $t$. Owing to the intensity dependence of the refractive index, each part of the pulse will experience its own additional phase shift $\Delta \phi$ on exiting the fibre, given by

$$
\Delta \phi=(2 \pi / \lambda) n_{2} L|E(t)|^{2}
$$

Spectral broadening of the pulse is a consequence of the time dependence of $\Delta \phi$ (Fig. 2), where frequencies in the leading half of the pulse are lowered, while those in the trailing are raised. If such a pulse

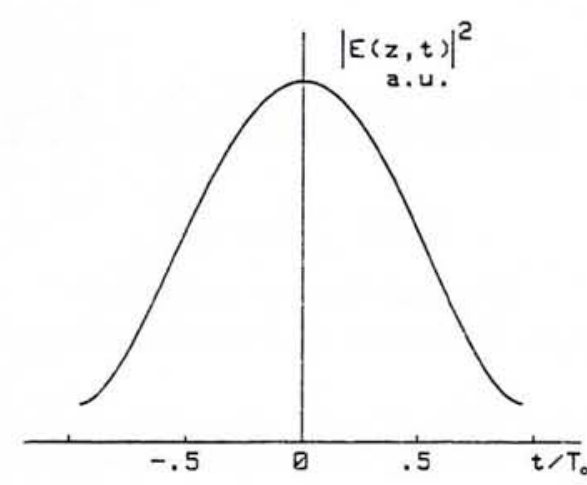

$\tau=T_{0}^{-1}(t-z \partial k / \partial \omega) ; \xi=\left|\partial^{2} k / \partial \omega^{2}\right| z T_{0}^{-2} ;$ $V=T_{0}\left(k /\left|\partial^{2} k / \partial \omega^{2}\right|^{2}\right) \tilde{E}$

For negative GVD $\left(\partial^{2} k / \partial \omega^{2}<0\right)$, Eq. 1 has the form:

$$
i \frac{\partial V}{\partial \xi}=\frac{1}{2} \frac{\partial^{2} V}{\partial \tau^{2}}+|V|^{2} V
$$

The terms on the right side describe the effects of GVD and of the nonlinear refractive index, respectively: optical losses in the medium are not accounted for.

Zakharov and Shabat showed analytically that the equation has soliton solutions given by

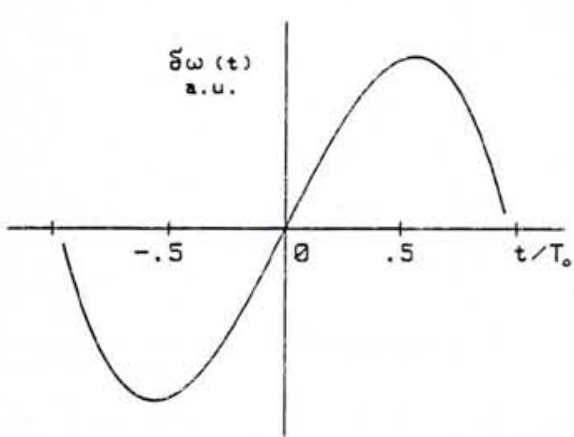

Fig. 2 - Envelope of a laser pulse. a, upper) The square of the envelope of the intensity $|E(z, t)|$ of a laser pulse plotted as a function of the normalised time $t / T_{0}$ showing spectral broadening in a glass fibre.

$b$, lower) Spectral broadening (frequency chirp) of the pulse: frequencies in the trailing half are lowered, those in the leading half raised. If broadening due to dispersion is balanced by narrowing due to non-linearity, the pulse propagates down the lossless fibre without a change in shape.

travels through a medium with negative GVD, as for silica fibres at $\lambda>1.3 \mu \mathrm{m}$, the trailing half of the pulse with higher frequencies will propagate at a higher velocity than the leading half with lower frequencies: the result is pulse narrowing.

Such behaviour for the pulse envelope shape allows one to suppose that the optical pulse in a fibre is a soliton, especially if the shape broadening due to dispersion is exactly balanced by narrowing due to nonlinearity. For in this case the pulse will propagate down the lossless fibre without changing shape.

\section{Picosecond Solitons}

Evolution of the envelope $E(z, t)$ shape of a long $\left(T_{0}>1 \mathrm{ps}\right)$ optical pulse $\widetilde{E}(z, t)=$ $\widetilde{E}(z, t) \exp \left[i\left(\omega_{0} t-k_{0} z\right)\right]$ in a nonlinear dispersive medium in the $z$ direction is described by the nonlinear Schrödinger equation

$$
i \frac{\partial \tilde{E}}{\partial z}+\frac{\partial k}{\partial \omega} \frac{\partial E}{\partial t}=\frac{-1}{2} \frac{\partial^{2} K}{\partial \omega^{2}} \frac{\partial^{2} \tilde{E}}{\partial t^{2}}+k|\tilde{E}|^{2} \tilde{E}
$$

where $k=k_{0} n^{2} / 2 n_{0}$ and $k_{0}=2 \pi / \lambda$ and $\omega$ is the optical frequency. The equation is reduced to the dimensionless form by the transformation
$V(\xi, \tau)=$

$æ \operatorname{Sech}[æ(\tau-v \xi)] \exp \left[-i v t+i\left(v^{2}-æ^{2}\right) \xi / 2\right]$

where $\nsim$ is a soliton form factor determining the soliton amplitude and duration and $v$ is the soliton velocity. The fundamental soliton ( $N=1)$ propagates along the lossless fibre without change in shape for arbitrarily long distances - a property that makes it attractive for data transmission in optical fibre communication systems.

The peak power $P_{1}$ required to support the fundamental soliton is given by $P_{1}=$ $\left|\beta_{2}\right| \lambda A_{\text {eff }} / n_{2} T_{0}^{2}$ where $T_{0}$ is the pulse width, $\beta_{2}=\left(\lambda^{3} / 2 \pi c^{2}\right) \mathrm{d}^{2} n / \mathrm{d} \lambda^{2}$ is the GVD parameter and $A_{\text {eff }}$ is the effective fibre core cross-sectional area. The latest single-mode silica fibres typically have $P_{1} \approx$ $1 \mathrm{~mW}$ for $T_{0}=30 \mathrm{ps}$ at $\lambda=1.55 \mu \mathrm{m}$, implying that the radiation power from semiconductor lasers is sufficient to launch fundamental solitons into the fibres.

The peak power necessary to excite the $N$-th order soliton is $N^{2}$ times that required for the fundamental soliton. The evolution of the pulse intensity envelope shape for higher-order solitons can be obtained by solving numerically the nonlinear Schrödinger equation. It is found that the behaviour of higher-order solitons propagating along a fibre is rather complex: the pulses undergo narrowing and splitting, but revert to their original shape at a length equal to the soliton period $Z_{0}$ $=(\pi / 2) T_{0}^{2} /\left|\beta_{2}\right|$. The second-order soliton $(N=2)$ has the simplest behaviour: the pulse narrows to a minimum width at the half period then broadens to recover its shape at the full soliton period. Fig. 3 shows the evolution of the third-order soliton over one soliton period. It is seen, that the pulse first contracts to a minimum width at about $Z_{0} / 4$ and then splits into two equal pulses at $Z_{0} / 2$ before merging again to adopt a minimum width at $3 Z_{0} / 4$, and finally regaining the original shape at $Z=Z_{0}$.

\section{Experimental verification}

Mollenauer, Stolen and Gordon in 1980 were the first to verify experimentally the behaviour predicted previously for solitons. They observed, as a function of the input pulse power, the shapes of pulses 
Fig. 3 - Calculated behaviour over one soliton period $z_{0}$ for the third-order soliton: the dimensionless intensity envelope is plotted as a function of time normalised to the pulse width $T_{0}$.

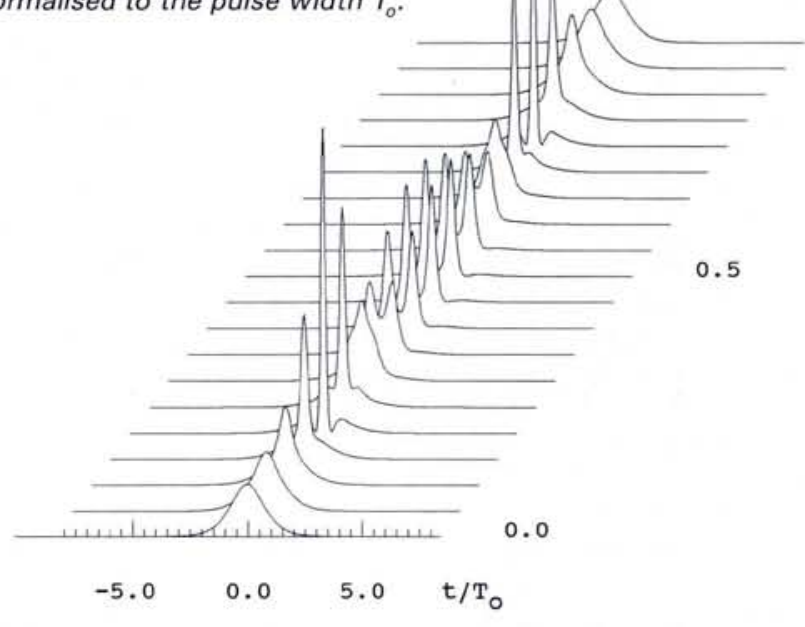

$\mathrm{z} / \mathrm{z}_{\mathrm{o}}$

1.0

Fig. 4-Measured autocorrelation shapes (intensity versus time) of laser pulses, for five different input peak powers $P_{0}$, measured over one-half the soliton period at the output of a silica glass fibre. Shown inset in the upper part are the spectrum (left) and autocorrelation shape (right) of the input laser pulse.

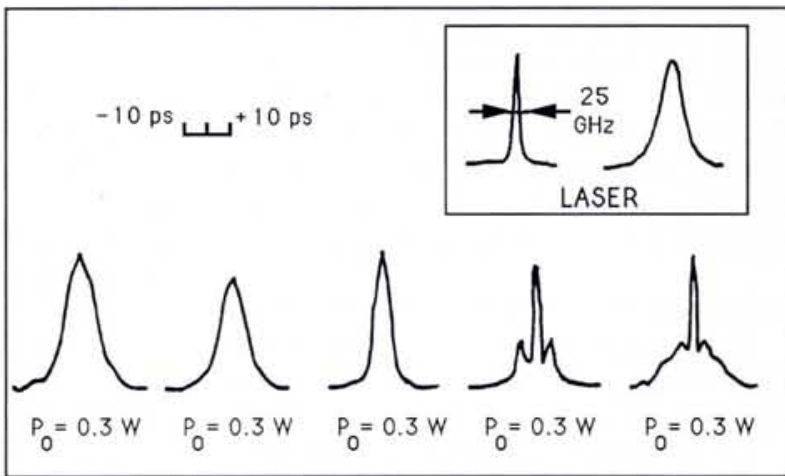

using an autocorrelation technique at the fibre output. A mode-locked colour-centre laser was used to obtain 7 ps optical pulses near $\lambda=1.55 \mu \mathrm{m}$, a wavelength at which losses in the fibre are at a minimum and fibre dispersion is negative. In one experiment, the peak power was varied over the range $0.3-25 \mathrm{~W}$ and the pulse shape was measured at the output of a $700 \mathrm{~m}$ long single-mode fibre (the length being about one-half the soliton period). The calculated value of the fundamental soliton peak power $P_{1}$ for a 7 ps optical pulse and the fibre employed was $1.0 \mathrm{~W}$.

The lower part of Fig. 4 shows the measured autocorrelation shapes of pulses at the output of the fibre for five input peak powers (the autocorrelation shape and the frequency spectrum of laser pulses launched into the fibre are shown for comparison in the upper part). It is seen that at a low power $(0.3 \mathrm{~W})$, the pulse experiences dispersion broadening: the nonlinear effect is small at this power level. At the input power $P_{0}=1.2 \mathrm{~W}$ corresponding approximately to the fundamental soliton power, dispersion broadening of the pulse is balanced by nonlinear narrowing and the fibre output pulse shape coincides with the input one. At $P_{0}=5$ $W$, the pulse narrows to the minimum width corresponding to the half-period behaviour of the second-order solitons. The power $P_{\mathrm{o}}=11.4 \mathrm{~W}$ corresponds approximately to the third-order solitons and here the autocorrelation curve shows well-resolved pulse splitting. As three-fold splitting in the autocorrelation curve corresponds to a two-fold splitting of the pulse itself, we have good agreement with the predicted behaviour for third-order solitons (Fig. 3). The observed five-peak structure of the autocorrelation trace for $P_{\text {o }}=22.5 \mathrm{~W}$ corresponds to a three-fold splitting of the pulse - also agreeing with predictions of the Schrödinger equation (Eq. 1) for the fourth-order soliton.
It has been already mentioned that higher-order solitons should regain their original shapes at a distance $Z=Z_{0}$ (or multiples of $Z_{0}$ ). Such a restoration was demonstrated for second-order and thirdorder solitons by Stolen and Mollenauer. In contrast to the previous experiment, they used a fibre length of $1.3 \mathrm{~km}$ corresponding to about one soliton period.

\section{Soliton compression}

The compression of higher-order soliton pulses to a minimum width at fibre lengths of only a fraction of a soliton period (Fig. 3) has become a common method for producing ultrashort pulses (soliton compression). The compression factor $F_{\mathrm{c}}$ and the optimum fibre length $Z_{\text {opt }}$ of such compressors are given approximately by the empirical relations

$$
\begin{aligned}
& F_{\mathrm{c}}=\tau_{\text {input }} / \tau_{\mathrm{c}} \approx 4.1 \mathrm{~N} ; \\
& z_{\text {opt }} / z_{\mathrm{o}} \approx 0.32 / \mathrm{N}+1.1 / \mathrm{N}^{2}
\end{aligned}
$$

The soliton compressor operates in the wavelength range $1.3-1.6 \mu \mathrm{m}$ where the GVD is negative. In the visible and nearinfrared regions, where the GVD is positive, a fibre is used only to impose spectral broadening on the pulse through a selfphase modulation effect. The spectrally broadened ("chirped") pulse is then compressed externally using a grating pair which provides negative GVD (fibre grating compression).

Both types of compression have been used to generate femtosecond pulses. Grudinin, Dianov et al. (General Physics Institute, Moscow) and Mitschke and Mollenauer (Bell Labs, USA) produced 18 fs pulses at $=1.6 \mu \mathrm{m}$ using soliton compression; Fork et al. obtained 6 fs pulses at $=0.62 \mu \mathrm{m}$ with the fibre grating compression technique. The pulses consisted of about three optical cycles in both cases.

\section{Sub-picosecond and Femtosecond Solitons}

As the pulse length $T_{0}$ is reduced, the spectral width $\Delta f$ broadens as $T^{-1}$. For $T_{0}=100 \mathrm{fs}, \Delta f$ is about $10^{13} \mathrm{~Hz}$ for a carrier frequency $f \approx 2 \times 10^{14} \mathrm{~Hz}(\lambda=$ $1.5 \mu \mathrm{m})$. The large value of the pulse spectrum suggests that one needs to account for higher-order GVD, the dispersion of the nonlinearity and a finite response time of the nonlinearity in order to describe the propagation of sub-picosecond pulses through fibres. Numerical solutions of the nonlinear Schrödinger equation (modified by including high-order dispersive and nonlinear terms) as well as investigations of the propagation of subpicosecond pulses in fibres have revealed a number of new effects. In particular, soliton decay occurs where sub-picosecond higher-order solitons decompose into their constituents: second-order solitons decay into two solitons, each propagating at different speeds; third and higher-order solitons decay in a similar fashion.

Another remarkable effect is connected with the finite (delayed) response time of the nonlinearity. This delayed response is observed as a continuous redshift in the mean frequency of sub-picosecond soliton pulses propagating in optical fibres. As mentioned earlier, the delayed nonlinear response gives rise to Raman effects. Physically, the frequency shift of pulses can be explained in terms of the Raman effect as follows: silica glass exhibits a broad Raman gain spectrum stretching from about zero detuning to more than $1000 \mathrm{~cm}^{-1}$. The spectral width of subpicosecond pulses is large enough for the low frequency side of the pulse spectrum to provide a seed for Raman amplification, i.e., the higher frequency components of the pulse pump the lower frequency components through self-induced stimulated 
Raman scattering. Energy from blue components is thereby continuously transferred to the red components leading to a decrease in the mean frequency of pulses.

The redshift phenomenon was discovered experimentally by Dianov, Mamyshev et al. and by Mitschke and Mollenauer. In a typical experiment, a frequency shift of $8 \mathrm{THz}$ was observed when $500 \mathrm{fs}$ pulses were propagated through a $392 \mathrm{~m}$ long single-mode fibre. It was found that the frequency shift depended on the pulse width as $T_{0}^{-4}$. A theory has been developed by Gordon and the phenomenon should be allowed for when considering the propagation and amplification of subpicosecond and femtosecond soliton.

\section{Soliton-Based Communication Systems}

The prospects for using solitons in communication systems have stimulated many investigations of practical aspects.

A soliton laser developed by Mollenauer and Stolen plays an important rôle in the study of soliton propagation through fibres and has become a useful experimental tool. It comprises a synchronously pumped, mode-locked, colour-centre laser, whose cavity is coupled to second cavity containing a piece of single-mode, polarization-preserving fibre. The fibre is used in a laser feed-back loop to shape stable, bandwidth-limited pulses. The soliton laser produces a sequence of short pulses at the wavelength of $1.5 \mu \mathrm{m}$ at a repetition rate of $100 \mathrm{MHz}$. The pulse width depends on the fibre length and can be changed in the range $\approx 0.1-2 \mathrm{ps}$.

However, semiconductor lasers remain the most promising soliton sources for operating systems. Even if the initial pulse shape or the peak power differs considerably from the equivalent parameter for a fundamental soliton propagating through a fibre, pulses will eventually evolve into exact solitons with widths depending on the input power.

\section{Fibre losses}

Losses in the fibre result in the soliton peak power decreasing with the propagation distance. This, in turn, leads to an increase in the width of the fundamental soliton which can limit the transmission rate. However, the peak power of a soliton can be increased over a very small time interval - a remarkable feature that allows the detrimental influence of fibre losses on soliton transmission to be overcome using periodic optical amplification.

A comprehensive investigation of optical soliton amplifiers has been conducted recently. Systems included semiconductor, Raman and Er-doped fibre amplifiers. The Er-doped fibre amplifier comprising an Er-doped silica fibre pumped by semiconductor laser radiation is presently considered the most promising for application in soliton communication systems. Its main advantage is a wide bandwidth, high gain, low coupling losses, and the ability to amplify signals at $\lambda=1.5 \mu \mathrm{m}$.

\section{Soliton interaction}

The interaction between neighbouring solitons can limit the transmission rate as the bit rate of a communication system is determined by the time interval between pulses. Interactions involving the direct overlap of pulses have been studied and it was found that the interaction force between neighbouring solitons decreases exponentially with their separation, and changes sinusoidally as a function of the relative phases. Solitons are also influenced by other factors such as the initial frequency spectrum broadening (chirp) of pulses, higher-order nonlinear and dispersive terms, etc. A pulse separation of $\approx 10$ pulse widths was found to be sufficient to allow short-range interactions between solitons to be neglected.

However, Smith and Molenauer have recently discovered an interaction between two periodic trains of 55 ps solitons shifted in time by several dozens of soliton widths - a so-called long-range interaction - a theory for which has been developed in the General Physics Institute, Moscow, and is in good agreement with the experimental results. According to this theory, an optical soliton excites, through electrostriction, acoustic waves which propagate in a direction transverse to the fibre axis. This leads to a temporal perturbation of the fibre's refractive index and trailing optical solitons become phase modulated and change carrier frequency. Overall, there is an additional shift in time of the solitons, i.e., the solitons interact.

Long-range soliton interaction decreases strongly on decreasing the fibre dispersion (as the square of the dispersion). Dispersion must nevertheless be taken into account when estimating the ultimate bandwidth of a soliton communication system.

\section{Soliton transmission experiments}

Successful experiments on soliton data transmission over distances above 100 $\mathrm{km}$ have been carried in a number of coun- tries in recent years. Semiconductor lasers were used as the soliton source and the solitons were amplified periodically using Er-doped fibre amplifiers: the soliton width lay in the range $10-75 \mathrm{ps}$ and the transmission rate at some $4-20 \mathrm{Gbit} / \mathrm{s}$.

It is worthwhile to note the experiment by Mollenauer et al. in which 50 ps solitons were transmitted in a $75 \mathrm{~km}$ recirculating loop consisting of three $25 \mathrm{~km}$ lengths of fibre interspersed with three $2.2 \mathrm{~m}$ long Er-doped fibre amplifiers. They achieved transmission over $9000 \mathrm{~km}$ for bit rates up to $4 \mathrm{Gbit} / \mathrm{s}$, thus demonstrating the practicality of essentially errorfree transmission over trans-oceanic distances at multigigabit per second rates.

\section{Conclusions}

Ten years have passed since the first experimental demonstration of the propagation of optical solitons in low-loss fibres. Many substantial scientific investigations mounted since then in different countries have resulted in viable solitonbased communication systems. It seems likely that another ten years will see optical solitons carrying information in a fibreoptic loop around the world.

\section{ACKNOWLEDGEMENT}

The author would like to thank Professor A.M. Prokhorov for his enthusiastic support of the investigation of optical solitons in fibres.

\section{FURTHER READING}

Mollenauer L.F. and Stolen R.H., Laser Focus (April 1982) 193.

Doran N.J. and Blow K.J., IEEE J. Quantum. Electron. QE-19 (1983) 1883.

Dianov E.M. et al., Nonlinear Effects in Optical Fibers, Laser Science and Technology An International Handbook 6, Eds.: V.S. Letokov et al. (Harwood Academic Publishers) 1989.

Agrawal G.P., Nonlinear Fiber Optics (Academic Press) 1989.

Smith K. and Mollenauer L.F., Opt. Lett. 14 (1989) 1284.

Dianov E.M. et al., Soviet Lightwave Comm. 1 (1991) 235.

Mollenauer L.F. et al., Opt. Lett. 15 (1990) 1203.

\section{UNIVERSITY OF AVEIRO}

\section{POST-DOCTORAL POSITION}

We expect to open a post-doctoral position from October 1992 to October 1994.

The applicant is expected to conduct experimental work in the field of Defects in Semiconductors. The main experimental technique is EPR. FTIR spectroscopy is also available. A Ph.D. degree is required and experience in the area will be considered. To apply, send a curriculum vitae with copies of publications and the names of three referees to:
Prof. M.H. Nazaré

Departamento de Física

Universidade de Aveiro P-3800 Aveiro, Portugal 